\title{
Review
}

\section{Soluble Form of the Triggering Receptor Expressed on Myeloid Cells-1 as a Marker of Microbial Infection}

\author{
Sébastien Gibot, MD, Service de Réanimation Médicale, Nancy, France \\ Aurélie Cravoisy, MD, Service de Réanimation Médicale, Nancy, France
}

\begin{abstract}
Sepsis is a common cause of morbidity and mortality in intensive care units. Unfortunately, there is no gold standard for diagnosing sepsis as clinical and laboratory signs are neither sensitive enough nor specific enough, and microbiological studies often remain negative. Thus, there is a need for clinical or laboratory tools to distinguish between sepsis and non-infectious inflammatory disorders. Among the potentially useful markers of sepsis, procalcitonin has been suggested to be the most promising, although results are variable depending on the severity of illness and degree of infection in the patient population studied. The triggering receptor expressed on myeloid cells-1 (TREM-1) is a member of the immunoglobulin superfamily, of which expression is upregulated on phagocytic cells in the presence of bacteria or fungi. Here we report on the potential usefulness of assessing the soluble form of TREM-1 (sTREM-1) in biological fluids for the diagnosis of microbial infection.
\end{abstract}

RECEIVED: MAY 5, 2004

REPRINT REQUESTS:

Sébastien Gibot, MD

Service de Réanimation Médicale

29 avenue du Maréchal de Latttre de Tassigny

54000 Nancy, France

Telephone: +33-3-83852970

Fax: +33-3-83858511

Email: s.gibot@chu-nancy.fr
REVISED AND ACCEPTED: JULY 16, 2004

KEYWORDS:

Sepsis; Infection; Community-acquired infections;

Membrane glycoproteins;

C-reactive protein; Protein precursors;

Predictive value of tests;

Inflammation/diagnosis

GRANT SUPPORT:

Programme Hospitalier de Recherche Clinique 


\section{INTRODUCTION}

Sepsis is a common cause of morbidity and mortality in intensive care units (ICUs). ${ }^{1}$ Clinical and laboratory signs of systemic inflammation including changes in body temperature, tachycardia, and leukocytosis are neither sensitive enough nor specific enough for the diagnosis of sepsis and can often be misleading. Trauma, burns, pancreatitis, major surgery, and many other conditions may elicit clinical signs of systemic inflammatory response syndrome (SIRS) in the absence of microbial infection. ${ }^{2-4}$ Unfortunately, there is no gold standard for diagnosing sepsis, as cultures may be negative especially in cases of antibiotic pre-treatment or inadequate sampling. Indeed, more than $30 \%$ of infected patients remain without clear documentation of microbial infection. Moreover, results of microbiological studies are not immediately available. Clinicians often feel uncomfortable about the diagnosis and may administer unneeded antibiotics while awaiting laboratory results. The empirical use of broad-spectrum antibiotics in patients without infection is potentially harmful, facilitating colonization and superinfection with multidrug resistant bacteria. ${ }^{5}$

There is a need for clinical or laboratory tools capable of making the distinction between SIRS and sepsis. Among the potentially useful markers of sepsis, procalcitonin has been suggested to be the most promising. ${ }^{6-8}$ However, several investigators have questioned the diagnostic and prognostic accuracy of routine procalcitonin measurements, reporting inconsistent and variable results depending on the severity of illness and degree of infection in the patient population studied. ${ }^{9-11}$

The triggering receptor expressed on myeloid cells- 1 (TREM-1) is a recently discovered member of the immunoglobulin superfamily. ${ }^{12}$ Like other similar cell-surface receptors, TREM-1 has a short intracellular domain and, when bound to its as yet unidentified ligand, associates with a signal-transduction molecule, DAP12, triggering the secretion of inflammatory cytokines that amplify the host response to microbial agents. ${ }^{12,13}$ Studies have shown that the expression of TREM-1 is greatly upregulated in the presence of bacteria, such as Pseudomonas aeruginosa and Staphylococcus aureus, or fungi such as Aspergillus fumigatus, in cell culture, peritoneal lavage fluid, and tissue samples from patients infected with these microorganisms. ${ }^{12,13}$ Conversely, TREM-1 is not upregulated in samples from patients with non-infectious inflammatory conditions like psoriasis, ulcerative colitis, or immune-complex mediated vasculitis, defining the specific involvement of this receptor only in cases of infection. ${ }^{13}$ Together with an upregulation of expression of the membrane-bound TREM-1, a soluble form (sTREM-1) may be released during infection.

\section{PLASMA LEVELS OF STREM-1 FOR THE DIAGNOSIS OF HUMAN SEPSIS}

The diagnostic value of the plasma sTREM-1 assay in distinguishing sepsis from severe systemic non-infectious inflammation among newly admitted critically ill patients with suspected infection was prospectively investigated. ${ }^{14}$ Consecutive patients newly hospitalized in a teaching hospital ICU in France between July 2003 and September 2003 were prospectively enrolled in the study if they had a clinically suspected infection and fulfilled at least two criteria of SIRS. ${ }^{15}$ Clinically suspected infection was defined as an explicit statement by the attending physician indicating the suspicion of an ongoing infection combined with the initiation of a diagnostic evaluation to identify or rule out infection and the subsequent prescription of antimicrobial therapy. Upon admission into the ICU, the following items were recorded for each enrolled patient: age, sex, severity of underlying medical condition stratified according to the criteria of McCabe and Jackson, ${ }^{16}$ simplified acute physiology score II (SAPS II), ${ }^{17}$ Sepsis-related Organ Failure Assessment (SOFA) score (range 0 to 24, with scores for each organ system [respiration, coagulation, liver, cardiovascular, central nervous system, and kidney] ranging from 0 [normal] to 4 [most abnormal]), ${ }^{18}$ reason for admission to the ICU, principal diagnosis, vital signs, respiratory parameters, routine blood tests, and microbiologic culture results. Within 12 hours of admission and enrollment in the study, plasma procalcitonin concentrations were measured and plasma sTREM-1 levels assessed as previously described. ${ }^{14}$ Survival or death in the ICU was assessed during a follow-up period as long as 28 days. The attending physician was allowed to prescribe microbiologic tests and antimicrobial therapy according to the usual practice of the ICU without interference by the research team. Two intensivists retrospectively reviewed all medical records pertaining to each patient and independently classified the diagnosis as SIRS, sepsis, severe sepsis, or septic shock at the time of admission, according to established consensus definitions. ${ }^{15}$ Both intensivists were blinded to the results of plasma sTREM-1 values.

The baseline characteristics of the overall study group are shown in table 1 . The mean ( \pm standard deviation $[\mathrm{SD}]$ ) SAPS II and SOFA scores were $50.5( \pm 22.6)$ and $8.3( \pm 4.5)$, respectively. The ICU mortality rate of $26.3 \%$ was in agreement with the predictive risk of death based on the SAPSII score. Diagnosis was established as SIRS in 29 patients $(38 \%)$, sepsis or severe sepsis (grouped as 'sepsis') in 22 patients $(29 \%)$, and septic shock in 25 patients $(33 \%)$. Causative conditions of SIRS were as follows: cardiac surgery $(n=6)$, cardiogenic shock $(n=5)$, acute exacerbation of chronic obstructive pulmonary disease $(n=5)$, acute pancreatitis $(n=3)$, heat stroke $(n=3)$, gastrointestinal hemorrhage $(n=2)$, trauma $(n=1)$, and unknown $(n=4)$. Clinical characteristics did not differ significantly at inclusion between septic and non-septic patients (table 1). Infections were demonstrated to be of microbiological origin in 40 out of 49 infected patients with $55 \%$ being Gram-negative and $42 \%$ Gram-positive bacterial infections, and 3\% fungal infections. The major sources of infection were in the respiratory tract $(55 \%)$ and abdomen (22\%). Twenty-four percent of infected patients had a documented bloodstream infection. 
Table 1. Clinical and biological data at admission and outcome of patients. ${ }^{*}$

\begin{tabular}{|c|c|c|c|c|}
\hline Characteristic & Total $(n=76)$ & Septic patients $(n=47)$ & Non-septic patients $(n=29)$ & $P$ value \\
\hline Age, years & $60 \pm 15$ & $61 \pm 14$ & $59 \pm 15$ & 0.55 \\
\hline Sex ${ }^{\dagger}$ & & & & 0.06 \\
\hline Male & $54(71)$ & $37(79)$ & $17(59)$ & \\
\hline Female & $22(29)$ & $10(21)$ & $12(41)$ & \\
\hline McCabe & $1.3 \pm 0.8$ & $1.3 \pm 0.8$ & $1.3 \pm 0.9$ & 0.57 \\
\hline SAPS II & $50.5 \pm 22.6$ & $52.6 \pm 23.8$ & $46.5 \pm 20.5$ & 0.65 \\
\hline SOFA score & $8.3 \pm 4.5$ & $9.7 \pm 4.8$ & $5.8 \pm 2.6$ & 0.38 \\
\hline Temperature $\left[{ }^{\circ} \mathrm{C}\right]$ & $37.9 \pm 1.0$ & $37.9 \pm 1.1$ & $37.9 \pm 1.0$ & 0.38 \\
\hline Leukocytes [g/l] & $14.4 \pm 7.6$ & $14.4 \pm 8.2$ & $13.9 \pm 3.8$ & 0.61 \\
\hline CRP [mg/l] & $154.1 \pm 142.8$ & $203.9 \pm 147.7$ & $62.7 \pm 65.3$ & 0.002 \\
\hline PCT [ng/ml] & $20.9 \pm 44.3$ & $31.4 \pm 52.4$ & $1.1 \pm 2.2$ & $<0.001$ \\
\hline sTREM-1 [ng/ml] & $112 \pm 953$ & $161 \pm 826$ & $22 \pm 341$ & $<0.001$ \\
\hline Mortality rate ${ }^{\dagger}$ & $20(26.3)$ & 15 (31.9) & 5 (17.2) & 0.16 \\
\hline \multicolumn{5}{|c|}{$\begin{array}{l}\text { * Values are expressed as the mean } \pm \text { standard deviation unless otherwise indicated. } P \text { values are for the comparison of septic vs. non-septic patients. Manr } \\
\text { Whitney } U \text { test for numerical data and Pearson } \chi^{2} \text { test for categorical data. } \\
{ }^{\dagger} \text { Values are expressed as number (percentage). } \\
\text { CRP, C-reactive protein; PCT, procalcitonin; SAPS II, Simplified Acute Physiology Score; SOFA, Sepsis-related Organ Failure Assessment; sTREM-1, soluble } \\
\text { triggering receptor expressed on myeloid cells-1. }\end{array}$} \\
\hline
\end{tabular}

Baseline plasma levels of C-reactive protein, procalcitonin, and sTREM-1 were higher among septic patients than among subjects with SIRS only (table 1, figure 1). Plasma sTREM-1 levels appeared to be most helpful in differentiating patients with sepsis from those with SIRS. Mean plasma sTREM-1 levels at admission were $22 \mathrm{ng} / \mathrm{ml}$ in SIRS, $183 \mathrm{ng} / \mathrm{ml}$ in sepsis, and $141 \mathrm{ng} / \mathrm{ml}$ in septic shock. As shown in figure 2, plasma sTREM-1 levels yielded the highest discriminative value with the area under the receiver-operating-characteristic (ROC) curve (AUC) being 0.97 followed by procalcitonin $(\mathrm{AUC}=0.85)$ and $\mathrm{C}$-reactive protein $(\mathrm{AUC}=0.77)$. At a cut-off of $60 \mathrm{ng} / \mathrm{ml}$, sTREM-1 yielded a sensitivity of $96 \%$ and a specificity of $89 \%$ in differentiating patients with SIRS from those with sepsis or septic shock.

\section{BRONCHOALVEOLAR STREM-1 IN THE DIAGNOSIS OF PNEUMONIA}

The diagnostic value of sTREM-1 in the context of a more localized infectious process, pneumonia, has also been investigated. ${ }^{19}$ Patients, 18 years or older, hospitalized in an ICU were prospectively enrolled if they met the following criteria: 1) need for mechanical ventilation and 2) clinical suspicion of bacterial pneumonia. Bacterial pneumonia was suspected if a newly developed and persistent infiltrate on chest radiography was associated with at least one of the following: purulent tracheal secretions, body temperature of at least $38.3^{\circ} \mathrm{C}$, and leukocytosis $(>10 \mathrm{~g} / \mathrm{l})$ or leukopenia $(<4 \mathrm{~g} / 1) .{ }^{20}$ Ventilator-associated pneumonia was defined by acquisition of the disease after 48 hours of mechanical ventilation. Mini-bronchoalveolar lavage (BAL) and microbiological specimen processing were performed. ${ }^{21,22}$ The concentration of bacteria considered significant for the potential diagnosis of pneumonia was greater than $10^{3}$ colony forming units (CFU)/ml of BAL fluid. ${ }^{21}$ Post hoc diagnosis of pneumonia was made from a combination of the clinical criteria and the microbiological evidence of bacterial infection. These criteria were similar to those used for ventilator-associated pneumonia described by Pugin and colleagues. $^{20}$ Pneumonia was considered to be absent when an alternative cause for pulmonary infiltrate was established and there was non-significant bacterial growth in the culture of BAL in association with a full recovery without antimicrobial therapy. Tumor necrosis factor (TNF)- $\alpha$, interleukin (IL)-1 $\beta$ and sTREM-1 concentrations were measured in BAL fluid.

Diagnosis was established as community-acquired pneumonia in $26 \%$ of enrolled patients, ventilator-associated pneumonia in $31 \%$, and no pneumonia in $43 \%$. Body temperature, leukocyte count, ratio of the partial pressure of arterial oxygen to the fraction of inspired oxygen $\left(\mathrm{PaO}_{2} / \mathrm{FiO}_{2}\right)$, serum $\mathrm{C}$-reactive protein and procalcitonin levels did not differ between the three groups (table 2). Bacterial species grew at a significant concentration from BAL $\left(>10^{3} \mathrm{CFU} / \mathrm{ml}\right)$ of all but two community-acquired pneumonia patients infected with Legionella pneumophila and all ventilator-associated pneumonia patients. The levels 

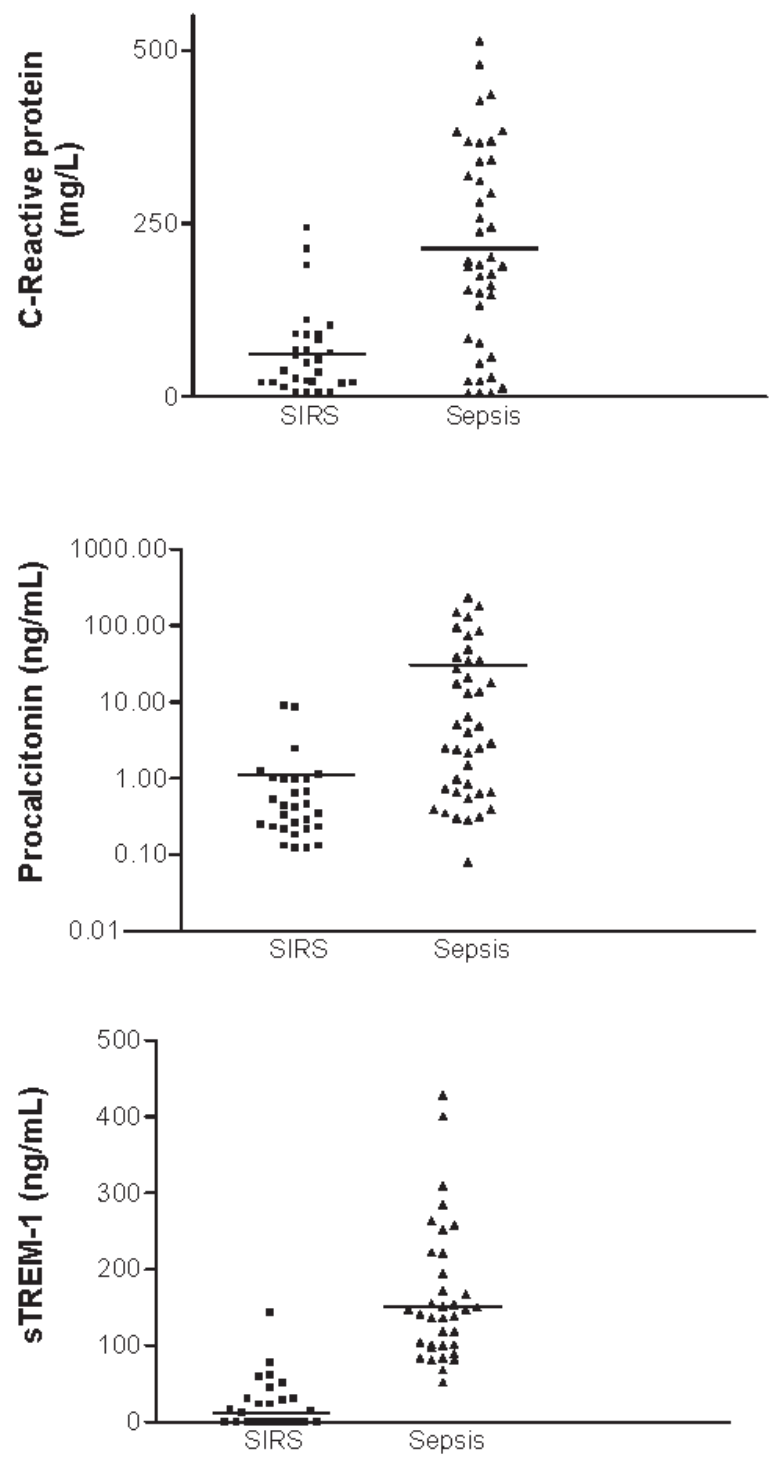

Figure 1. Plasma levels of C-reactive protein (CRP), procalcitonin (PCT) and sTREM-1 according to diagnosis upon admission to the ICU. Individual values are plotted. Horizontal bars represent the mean of the values. SIRS: patients with systemic inflammatory response syndrome of non-infectious origin $(n=29)$. Sepsis: patients with sepsis, severe sepsis or septic shock $(n=47)$. Baseline plasmatic levels of CRP, PCT and STREM-1 were higher among septic patients than among subjects with SIRS only (Mann Whitney $U$ test, all $P$ values $<0.002$ ). (Reprinted with permission. Gibot et al. Plasma level of a triggering receptor expressed on myeloid cells-1: its diagnostic accuracy in patients with suspected sepsis. Ann Int Med 2004;141:9-15. Copyright 2004 American College of Physicians. All rights reserved.) of sTREM-1 were higher in BAL fluid from

community-acquired and ventilator-associated pneumonia patients than from patients who did not have pneumonia, but did not differ significantly between community-acquired and ventilator-associated pneumonia patients (figure 3 ). TNF- $\alpha$ and IL-1 $\beta$ levels showed the same trend, but with a large overlap of values (figure 4). Soluble TREM-1 levels at or above $5 \mathrm{pg} / \mathrm{ml}$ were detected in BAL fluid from approximately $95 \%$ of community-acquired pneumonia patients, $100 \%$ of ventilator-associated pneumonia patients, and in approximately $10 \%$ of patients without pneumonia. Thus, among the enrolled patient population, the presence of sTREM-1 in BAL fluid is associated with a likelihood ratio of 10.38. The capacity of sTREM-1 to differentiate pneumonia from lack of pneumonia was assessed with a ROC curve analysis (figure 5). The area under the curve, when STREM-1 was used to differentiate pneumonia from lack of pneumonia, was 0.93 . A cut-off value of $5 \mathrm{pg} / \mathrm{ml}$ for sTREM-1 had a sensitivity of $98 \%$ and a specificity of $90 \%$.

\section{STREM-1: THE BIOLOGICAL MARKER OF INFECTION?}

The differentiation between infection, a microbiological insult, and sepsis, the host reaction to that microbial aggression, remains difficult. Infection can be present without sepsis, particularly in the debilitated and elderly, for example when blood cultures are positive without an accompanying sepsis syndrome. Alternatively, a sepsis-like response may be present without obvious infection, as in cases of trauma and major surgery. Although some patients will have clear evidence of infection, making diagnosis straightforward, microbiological data in many patients are equivocal or missing, and other diagnostic tools must be used. Elevated levels of many inflammatory mediators and

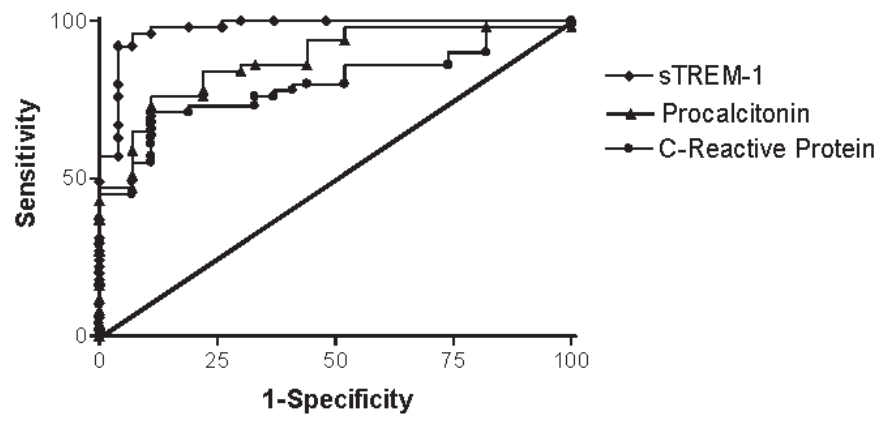

Figure 2. Receiver-operating-characteristic (ROC) curves for various cut-off levels of plasma C-reactive protein (CRP), procalcitonin (PCT) and STREM-1 in differentiating between presence and absence of infection. Areas under the ROC curves: 0.97 for sTREM-1, 0.85 for PCT, and 0.77 for CRP. At a cut-off of $60 \mathrm{ng} / \mathrm{ml}$, sTREM-1 correctly classifies patients as suffering from infection with $96 \%$ sensitivity and $89 \%$ specificity. (Reprinted with permission. Gibot et al. Plasma level of a triggering receptor expressed on myeloid cells-1: its diagnostic accuracy in patients with suspected sepsis. Ann Int Med 2004;141:9-15. Copyright 2004 American College of Physicians. All rights reserved.) 
Table 2. Characteristics of the three groups of patients at inclusion.*

\begin{tabular}{|c|c|c|c|c|}
\hline Characteristic & $\begin{array}{l}\text { nmunity-acquired } \\
\text { neumonia }(n=38)\end{array}$ & $\begin{array}{l}\text { Ventilator-associated } \\
\text { pneumonia }(n=46)\end{array}$ & $\begin{array}{l}\text { No pneumonia } \\
(n=64)\end{array}$ & $\boldsymbol{P}$ value $^{\dagger}$ \\
\hline $\begin{array}{l}\text { Duration of mechanical } \\
\text { ventilation before study } \\
\text { entry [days] }\end{array}$ & $0.4 \pm 0.2$ & $6.4 \pm 8.5$ & $2.1 \pm 4.8$ & $<0.001$ \\
\hline $\begin{array}{l}\text { Previous antimicrobial } \\
\text { therapy }[\mathrm{n}(\%)]\end{array}$ & $33(87)$ & $19(41)$ & $30(47)$ & $<0.001$ \\
\hline Shock [n (\%)] & $18(47)$ & $19(41)$ & $30(47)$ & 0.49 \\
\hline Body temperature $\left[{ }^{\circ} \mathrm{C}\right]$ & $37.9 \pm 2.0$ & $38.1 \pm 0.9$ & $37.7 \pm 1.1$ & 0.82 \\
\hline Leukocyte count $[\mathrm{g} / \mathrm{l}]$ & $12.8 \pm 7.9$ & $13.4 \pm 8.5$ & $12.5 \pm 5.8$ & 0.99 \\
\hline $\mathrm{PaO}_{2} / \mathrm{FiO}_{2}[\mathrm{mmHg}]$ & $181 \pm 80$ & $203 \pm 67$ & $206 \pm 91$ & 0.51 \\
\hline PCT [ng/ml] & $3.7 \pm 1.9$ & $2.6 \pm 0.8$ & $2.5 \pm 1.2$ & 0.58 \\
\hline CRP [mg/l] & $197 \pm 128$ & $184 \pm 108$ & $141 \pm 110$ & 0.34 \\
\hline BAL fluid TNF- $\alpha$ [pg/ml] & $298.2 \pm 47.7$ & $290.5 \pm 39.7$ & $147.2 \pm 25.1$ & $<0.001$ \\
\hline BAL fluid TNF-1 $\beta$ [pg/ml] & $92.5 \pm 22.5$ & $95.1 \pm 29.4$ & $41.5 \pm 12.5$ & $<0.001$ \\
\hline BAL fluid sTREM-1 [pg/ml] & $23.2 \pm 2.8$ & $33.6 \pm 5.1$ & $1.8 \pm 0.9$ & $<0.001$ \\
\hline
\end{tabular}

*Values are expressed as the mean \pm Standard Deviation unless otherwise indicated.

$\dagger P$ values are comparisons between community-acquired pneumonia, ventilator-associated pneumonia, and no pneumonia groups. Mann Whitney $U$ test for numerical data and Pearson $\chi^{2}$ test for categorical data.

$\mathrm{BAL}$, bronchoalveolar lavage; CRP, C-reactive protein; $\mathrm{PaO}_{2} / \mathrm{FiO}_{2}$, ratio of the partial pressure of arterial oxygen to the fraction of inspired oxygen; $\mathrm{PCT}$, procalcitonin; sTREM-1, soluble triggering receptor expressed on myeloid cells-1; TNF, tumor necrosis factor.

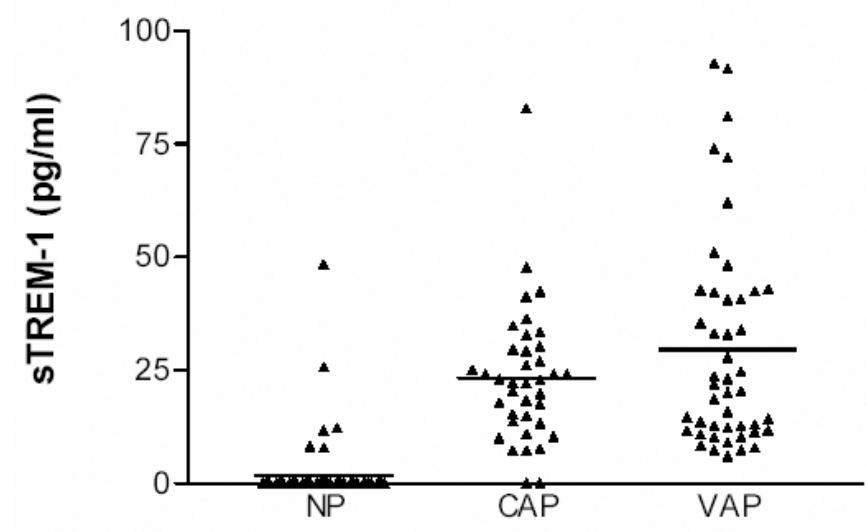

Figure 3. Levels of sTREM-1 in BAL fluid according to diagnosis. Individual values are plotted. Horizontal bars represent the mean of the values. NP, patients without pneumonia $(n=64)$. CAP, community-acquired pneumonia $(n=38)$. VAP, ventilator-associated pneumonia $(n=46)$. The levels of sTREM-1 were higher in BAL fluid from CAP and VAP patients than from NP patients (Mann Whitney $U$ test, $P<0.001$ ), but did not differ significantly between CAP and VAP patients. (Reprinted with permission. Gibot et al. Soluble triggering receptor expressed on myeloid cells and the diagnosis of pneumonia. N Engl J Med 2004 350:451-458. Copyright 2004 Massachusetts Medical Society. All rights reserved.)

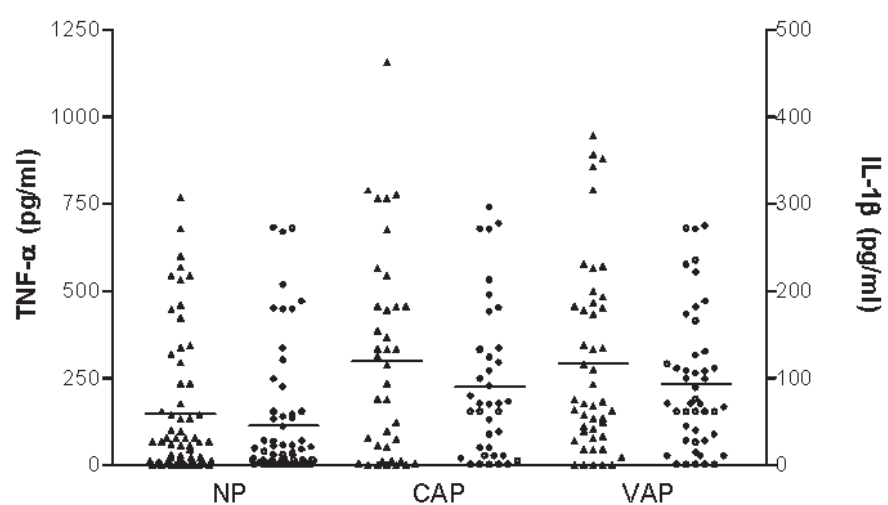

Figure 4. Levels of tumor necrosis factor- $\alpha$ (TNF- $\alpha$ ) and interleukin-1 $\beta$ (IL-1 $\beta$ ) in BAL fluid according to diagnosis. Individual values are plotted. Horizontal bars represent the mean of the values. TNF- $\beta$ and IL-1 $\beta$ levels were higher in BAL fluid from community-acquired pneumonia (CAP) and ventilator-associated pneumonia (VAP) patients than from patients without pneumonia (NP) (Mann Whitney $U$ test, $P<0.001)$, but did not differ significantly between CAP and VAP patients. 


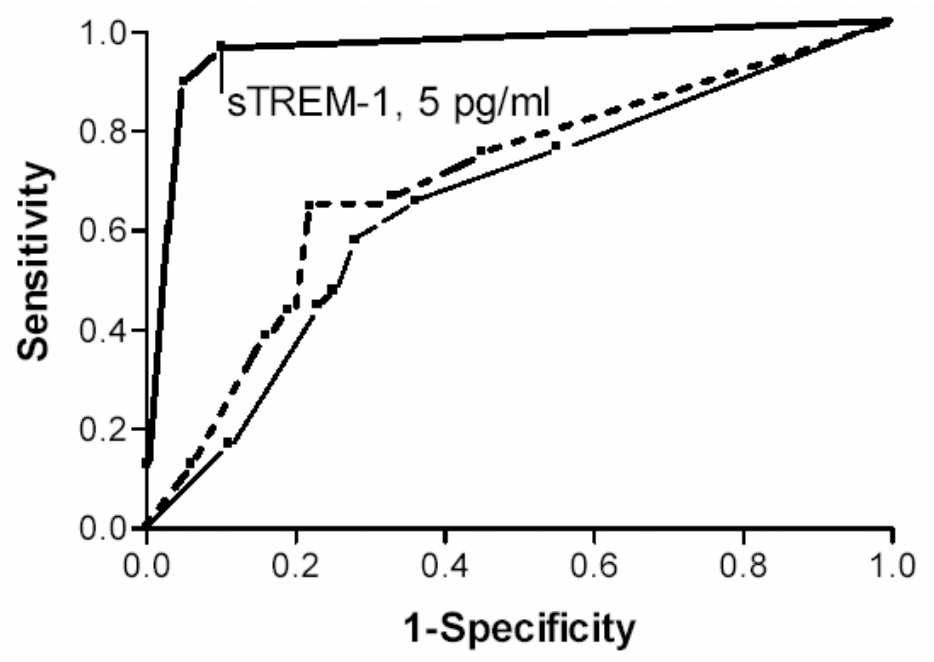

Figure 5. Receiver-operating-characteristic (ROC) curves for various cut-off levels of BAL fluid STREM-1, tumor necrosis factor- $\alpha$ (TNF- $\alpha$ ) and interleukin-1 $\beta$ (IL-1 $\beta$ ) in differentiating between presence and absence of pneumonia. Areas under the ROC curves: 0.93 for sTREM-1, 0.64 for TNF- $\alpha$, and 0.69 for IL-1 $\beta$. (Reprinted with permission. Gibot et al. Soluble triggering receptor expressed on myeloid cells and the diagnosis of pneumonia. N Engl J Med 2004 350:451-458. Copyright 2004 Massachusetts Medical Society. All rights reserved.)

Table 3. Performance of some biological markers of sepsis. ${ }^{10,24,25}$

\begin{tabular}{lcc}
\hline Marker $^{*}$ & Sensitivity, \% & Specificity, \% \\
\hline C3a & 86 & 80 \\
Interleukin-6 & 67 & 72 \\
Interleukin-8 & 63 & 78 \\
Procalcitonin & 67 to 97 & 61 to 78 \\
C-reactive protein & 72 & 67 \\
\hline
\end{tabular}

acute phase reactants have been described in sepsis, however none are specific enough for infection, as all can be increased in other inflammatory conditions without obvious infection (table 3 ).

The important point to consider in the use of clinical signs and biological markers to identify infection is that none should be used in isolation; a panel of indicators is of greater predictive value. For example, the evaluation of a combination of elevated cytokine levels has been shown to be more accurate than any single elevated cytokine level in determining patient survival. ${ }^{23}$ However, many tests, especially for cytokines, are very expensive, which may preclude their routine use. An ideal biological marker of sepsis would fulfill rigorous criteria: it must be specific and sensitive; it must be able to be rapidly performed; and it must be of low cost. The sTREM-1 assay seems, therefore, to be a good candidate. Its accuracy has been demonstrated both in pneumonia (98\% sensitivity, 90\% specificity) and in human sepsis (96\% sensitivity, 89\% specificity). Moreover, the specific involvement of TREM-1 during infection and not during non-infectious inflammatory disorders has been clearly evidenced by experimental studies. ${ }^{12,13}$ The technique used for its determination is rapid, inexpensive, and can be performed for small batches of specimens or individual samples. Therefore, sTREM-1 may constitute a reliable marker of infection particularly in plasma during sepsis ${ }^{14}$ and BAL fluid in cases of pneumonia. ${ }^{19}$ It remains to be determined whether sTREM-1 could be useful in other infectious conditions, such as infected ascites or meningitis. Obviously, the sTREM-1 assay does not determine the causative organism and thus does not preclude the clinician from performing microbiological studies. The real clinical value of the sTREM-1 assay is in allowing the clinician to withhold empiric antibiotics until culture results are available for cases where sTREM-1 levels are low, thus eliminating unnecessary antibiotic exposure to the patient. Finally, the usefulness of measuring sTREM-1 in clinical practice, especially for its potential impact on improvement of prognosis, has to be prospectively determined in dedicated studies.

\section{CONCLUSION}

The sTREM-1 assay appears to be a reliable marker of infection particularly during sepsis and pneumonia, though it remains to be determined whether sTREM-1 could be a useful marker of other infectious conditions. The real clinical value of the sTREM-1 assay comes when sTREM-1 levels are low, allowing the clinician to withhold empiric antibiotics until culture results are available, and thus eliminating unnecessary antibiotic exposure to the patient. Additional prospective studies will be necessary to determine the usefulness of measuring sTREM-1 in routine clinical practice. 


\section{REFERENCES}

1. Hotchkiss RS, Karl IE. The pathophysiology and treatment of sepsis. N Engl J Med 2003;348:138-150.

2. Reinhart K, Meisner M, Hartog C. Diagnosis of sepsis: Novel and conventional parameters. Adv Sepsis 2001;1:42-51.

3. Rangel-Frausto MS, Pittet D, Costigan M, Hwang T, Davis CS, Wenzel RP. The natural history of the systemic inflammatory response syndrome (SIRS). A prospective study. JAMA 1995;273:117-123.

4. Brun-Buisson C. The epidemiology of the systemic inflammatory response. Intensive Care Med 2000;26:S64-S74.

5. Amyes SG. Strategies and options for minimizing resistance emergence in pulmonary infections. Chest 1998;113:228S-232S.

6. Assicot M, Gendrel D, Carsin H, Raymond J, Guilbaud J, Bohuon C. High serum procalcitonin concentrations in patients with sepsis and infection. Lancet 1993;341:515-518.

7. Vincent JL. Procalcitonin: THE marker of sepsis? Crit Care Med 2000;28:1226-1228.

8. Brunkhorst FM, Wegscheider K, Forycki ZF, Brunkhorst R. Procalcitonin for early diagnosis and differentiation of SIRS, sepsis, severe sepsis, and septic shock. Intensive Care Med 2000;26:S148-S152.

9. Lapillonne A, Basson E, Monneret G, Bienvenu J, Salle BL. Lack of specificity of procalcitonin for sepsis diagnosis in premature infants. Lancet 1998;351:1211-1212.

10. Ugarte H, Silva E, Mercan D, De Mendonca A, Vincent JL. Procalcitonin used as a marker of infection in intensive care unit. Crit Care Med 1999;27:498-504.

11. Ruokonen E, Nousiainen T, Pulkki K, Takala J. Procalcitonin concentrations in patients with neutropenic fever. Eur J Clin Microbiol Infect Dis 1999;18:283-285.

12. Bouchon A, Dietrich J, Colonna M. Cutting edge: inflammatory responses can be triggered by TREM-1, a novel receptor expressed on neutrophils and monocytes. J Immunol 2000;164:4991-4995.

13. Bouchon A, Facchetti F, Weigand MA, Colonna M. TREM-1 amplifies inflammation and is a crucial mediator of septic shock. Nature 2001;410:1103-1107.

14. Gibot S, Kolopp-Sarda MN, Bene MC, Cravoisy A, Levy B, Faure GC, Bollaert PE. Plasma level of a triggering receptor expressed on myeloid cells-1: its diagnostic accuracy in patients with suspected sepsis. Ann Intern Med 2004;141:915.

15. Bone RC, Balk RA, Cerra FB, Dellinger RP, Fein AM, Knaus WA, Schein RM, Sibbald WJ. Definitions for sepsis and organ failure and guidelines for the use of innovative therapies in sepsis. The ACCP/SCCM Consensus Conference Committee. American College of Chest Physicians/Society of Critical Care Medicine. Chest 1992;101:1644-1655.

16. McCabe WR, Jackson GG. Gram-negative bacteremia. Arch Intern Med 1982;110:847-864.

17. Le Gall JR, Lemeshow S, Saulnier F. A new Simplified Acute Physiology Score (SAPS II) based on a European/North American multicenter study. JAMA 1993;270:2957-2963.

18. Vincent JL, Moreno R, Takala J, Willatts S, De Mendonca A, Bruining H, Reinhart CK, Suter PM, Thijs LG. The SOFA (Sepsis-related Organ Failure Assessment) score to describe organ dysfunction/failure. On behalf of the Working Group on Sepsis-Related Problems of the European Society of Intensive Care Medicine. Intensive Care Med 1996;22:707710.

19. Gibot S, Cravoisy A, Levy B, Béné MC, Faure G, Bollaert PE. Soluble triggering receptor expressed on myeloid cells and the diagnosis of pneumonia. N Engl J Med 2004 350:451458.
20. Pugin J, Auckenthaler R, Mili N, Janssens JP, Lew PD, Suter PM. Diagnosis of ventilator-associated pneumonia by bacteriologic analysis of bronchoscopic and non bronchoscopic "blind" bronchoalveolar lavage fluid. Am Rev Respir Dis 1991;143:1121-1129.

21. Papazian L, Thomas P, Garbe L, Guignon I, Thirion X, Charrel J, Bollet C, Fuentes P, Gouin F. Bronchoscopic or blind sampling techniques for the diagnosis of ventilator-associated pneumonia. Am J Respir Crit Care Med 1995;152:19821991.

22. Duflo F, Debon R, Monneret G, Bienvenu J, Chassard D, Allaouchiche B. Alveolar and serum procalcitonin: diagnostic and prognostic value in ventilator-associated pneumonia. Anesthesiology 2002;96:74-79.

23. Casey LC, Balk RA, Bone RC. Plasma cytokine and endotoxin levels correlate with survival in patients with the sepsis syndrome. Ann Intern Med 1993;119:771-778.

24. Selberg O, Hecker H, Martin M, Klos A, Bautsch W Kohl J. Discrimination of sepsis and systemic inflammatory response Syndrome by determination of circulating plasma concentrations of procalcitonin, protein complement $3 \mathrm{a}$, and interleukin-6. Crit Care Med 2000;28:2793-2798.

25. Harbarth S, Holeckova K, Froidevaux C, Pittet D, Ricou B, Grau GE, Vadas L. Pugin J; Geneva Sepsis Network. Diagnostic value of procalcitonin, interleukin-6, and interleukin-8 in critically ill patients admitted with suspected sepsis. Am J Respir Crit Care Med 2001;164:396: 402. 